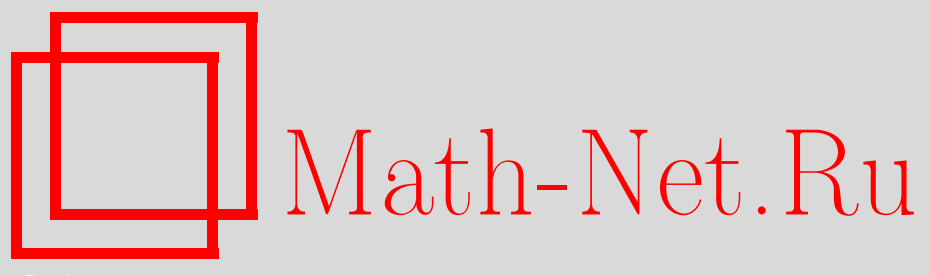

А. В. Политов, Критерий сходимости орторекурсивных разложений в евклидовых пространствах, Матем. заметки, 2013, том 93, выпуск 4, 637-640

DOI: https://doi.org/10.4213/mzm9263

Использование Общероссийского математического портала Math-Net.Ru подразумевает, что вы прочитали и согласны с пользовательским соглашением http://www . mathnet.ru/rus/agreement

Параметры загрузки:

IP : 52.6 .47 .48

26 апреля 2023 г., 15:20:05

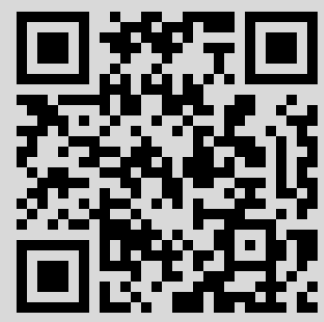




\section{Критерий сходимости орторекурсивных разложений в евклидовых пространствах}

\section{А. В. Политов}

1. Введение. Орторекурсивные разложения (далее ОРР) были введены Лукашенко [1]. Они являются естественным обобщением классических разложений элементов гильбертова пространства в ряды Фурье.

ОРР определяются следующим образом. Рассмотрим евклидово пространство $\mathcal{H}$ над полем действительных чисел и зафиксируем в нем произвольную систему элементов $\mathcal{E}=$ $\left\{e_{n}\right\}_{n=1}^{\infty}$ с единичной нормой. Для каждого элемента $f \in \mathcal{H}$ определим коэффициенты разложения. Положим $\widehat{f}_{1}=\left(f, e_{1}\right)$. Далее, если уже определены $\widehat{f}_{1}, \ldots, \widehat{f}_{n-1}$, положим

$$
\widehat{f}_{n}=\left(r_{n-1}(f), e_{n}\right), \quad \text { где } \quad r_{n-1}(f)=f-\sum_{k=1}^{n-1} \widehat{f}_{k} e_{k} .
$$

Коэффициенты $\left\{\widehat{f}_{n}\right\}_{n=1}^{\infty}$ называются орторекурсивными коэффициентами Фурье элемента $f$ по системе $\mathcal{E}$, ряд $\sum_{n=1}^{\infty} \widehat{f}_{n} e_{n}-$ орторекурсивным рядом Фуръе элемента $f$ по системе $\mathcal{E}$.

В случае, если система $\mathcal{E}$ является ортонормированным базисом, ОРР по ней совпадает с классическим разложением в ряд Фурье.

Для введенных разложений сохраняются такие свойства обычных рядов Фурье, как равенство Бесселя $\left\|r_{n}(f)\right\|^{2}=\|f\|^{2}-\sum_{k=1}^{n}\left|\widehat{f}_{k}\right|^{2}$ и неравенство Бесселя $\sum_{k=1}^{\infty}\left|\widehat{f}_{k}\right|^{2} \leqslant\|f\|^{2}$. Помимо этого, сходимость к разлагаемому элементу эквивалентна равенству Парсеваля $\sum_{k=1}^{\infty}\left|\widehat{f}_{k}\right|^{2}=\|f\|^{2}$. Доказательства этих утверждений см. в [1].

В 2007 г. Б. С. Кашиным был поставлен вопрос о получении критерия сходимости ОРР в терминах матрицы Грама системы $\mathcal{E}$. В случае, когда $\mathcal{H}=\mathbb{R}^{2}$, известен следующий критерий сходимости ОРP (см. [2]):

Теорема 1. Для сходимости орторекурсивного ряда Фуръе каждого элемента $f \in \mathcal{H}$ по системе $\mathcal{E} к$ разлагаемому элементу необходимо и достаточно выполнение равенства

$$
\prod_{n=1}^{\infty} g_{n, n+1}=0, \quad \text { əде } \quad g_{i j}=\left(e_{i}, e_{j}\right) .
$$

В настоящей работе получен ответ для случая произвольного пространства.

2. Критерий сходимости ОРР. Далее будем считать, что линейная оболочка системы $\mathcal{E}$ всюду плотна в $\mathcal{H}$. Вначале отметим следующий факт.

Лемма 1. Сходимость (слабая сходимость) орторекурсивного ряда Фуръе каждого элемента $f \in \mathcal{H}$ по системе $\mathcal{E} \kappa$ разлагаемому элементу равносильна сходимости (соответственно, слабой сходимости) орторекурсивного ряда Фуръе каждого элемента е $\in \mathcal{E}$ к разлагаемому элементу.

ДокАЗАТЕЛЬСТво. Утверждение леммы следует из равенства Бесселя, линейности ОРР и того, что линейная оболочка $\mathcal{E}$ всюду плотна в $\mathcal{H}$.

Работа выполнена при поддержке Российского фонда фундаментальных исследований (грант № 11-01-00476-a).

DOI: $10.4213 / \mathrm{mzm} 9263$

(C) А. В. Политов, 2013 
Введем несколько обозначений. Через $G$ обозначим матрицу Грама системы $\mathcal{E}$. Через $H$ обозначим матрицу, у которой элементы, лежащие не выше главной диагонали, совпадают с элементами $G$, а остальные равны 0 (т.е. $G=H+H^{\top}-I$, где $I$ - единичная матрица).

Для каждого $e_{n} \in \mathcal{E}$ рассмотрим его орторекурсивный ряд Фурье по системе $\mathcal{E}$, который будет иметь вид $\sum_{k=1}^{\infty} c_{n k} e_{k}$, где $c_{n k}=\widehat{\left(e_{n}\right)_{k}}$. Матрицу, состоящую из чисел $c_{n k}$ (т.е. матрицу, в $n$-й строке которой стоят орторекурсивные коэффициенты вектора $e_{n}$ ), обозначим через $C$.

Справедливы следующие утверждения.

Лемма 2. Сходимость орторекурсивного ряда Фуръе каждого элемента е $\in \mathcal{E} \kappa$ разлагаемому элементу равносильна равенству $C C^{\top}=G$.

Лемма 3. Слабая сходимость орторекурсивного ряда Фуръе каждого элемента из $\mathcal{E}$ к разлагаемому элементу эквивалентна равенству $C G=G$.

ДокАзАтельство леммы 2. Пусть $C C^{\top}=G$. Выражения, получающиеся из этого равенства для диагональных элементов матрицы $G$, представляют собой в точности равенства Парсеваля для каждого элемента из $\mathcal{E} ;$ следовательно, ОРР элементов $\mathcal{E}$ сходятся к разлагаемым элементам.

Пусть ОРР элементов из $\mathcal{E}$ сходятся к разлагаемым элементам. Тогда для них выполняется равенство Парсеваля, поэтому имеет место цепочка равенств

$$
\sum_{k=1}^{\infty} c_{n k} c_{l k}=\frac{1}{2}\left[\sum_{k=1}^{\infty}\left(c_{n k}+c_{l k}\right)^{2}-\sum_{k=1}^{\infty} c_{n k}^{2}-\sum_{k=1}^{\infty} c_{l k}^{2}\right]=\frac{1}{2}\left[\left\|e_{n}+e_{l}\right\|^{2}-2\right]=g_{n l} .
$$

Лемма доказана.

ДоКАЗАТЕЛЬСТво ЛЕммы 3. Утверждение следует из определения слабой сходимости и того, что линейная оболочка $\mathcal{E}$ всюду плотна в $\mathcal{H}$.

Эти критерии еще не решают поставленную задачу, поскольку содержат матрицу $C$, не выраженную через матрицу Грама. Выразим матрицу $C$ через матрицы $G$ и $H$.

Сначала отметим важные отличия в действиях над конечными и бесконечными матрицами (подробнее см. [3]). Во-первых, умножение бесконечных матриц не является ассоциативным. Во-вторых, матрица может иметь бесконечное количество обратных, кроме того, множества правых и левых обратных к данной матрице, вообще говоря, не совпадают. Для нижнетреугольных матриц с ненулевыми элементами на диагонали существует ровно одна правая обратная матрица, которая также будет являться одной из левых обратных. Алгоритм нахождения этой матрицы полностью аналогичен алгоритму нахождения обратной к треугольной матрице в конечном случае.

Обозначим через $H^{-1}$ правую обратную к $H$ матрицу. Имеет место следующая лемма.

Лемма 4. Справедливы равенства $G=C H^{\top}$ и $C=G\left(H^{-1}\right)^{\top}$.

ДокАЗАТЕЛЬСтво. Имеет место равенство

$$
c_{n k}=\left(r_{k-1}\left(e_{n}\right), e_{k}\right)=\left(e_{n}-\sum_{l=1}^{k-1} c_{n l} e_{l}, e_{k}\right)=g_{n k}-\sum_{l=1}^{k-1} c_{n l} g_{l k} .
$$

Так как $g_{k k}=1$, получаем, что $g_{n k}=\sum_{l=1}^{k} c_{n l} g_{l k}$. Переписывая это равенство в матричном виде, получаем, что $G=C H^{\top}$. Ввиду отсутствия ассоциативности для умножения бесконечных матриц второе равенство не следует напрямую из полученного, поэтому потребуются дополнительные рассуждения. Отметим, что далее в доказательстве леммы 4 используется только то, что матрица $H$ треугольная и на диагонали у нее ненулевые числа.

Обозначим через $G_{m}, H_{m}, C_{m}$ верхние левые блоки матриц $G, H, C$ соответственно, состоящие из элементов, индексы которых не превосходят $m$. Справедливо равенство 
$G_{m}=C_{m} H_{m}^{\top}$, откуда $C_{m}=G_{m}\left(H_{m}^{-1}\right)^{\top}$. Поскольку матрица $H$ треугольная, справедливо равенство $H_{m}^{-1}=\left(H^{-1}\right)_{m}$ (так как соответствующие линейные уравнения для нахождения элементов обратных матриц совпадают); следовательно, $C_{m}=G_{m}\left(H^{-1}\right)_{m}^{\top}$ для любого натурального $m$. Для произвольных $n, k$ возьмем $m=\max (n, k)$; тогда с учетом последнего равенства $c_{n k}=\sum_{l=1}^{k} g_{n l}\left(\left(H^{-1}\right)^{\top}\right)_{l k}$ или, в матричном виде, $C=G\left(H^{-1}\right)^{\top}$, что и требовалось.

Таким образом, матрица $C$ выражена через $G$ и $H$, и леммы 2,3 можно трактовать как критерии сходимости ОРР в терминах матрицы Грама. Так, критерий слабой сходимости приобретает следующий вид.

Теорема 2. Следующие утверждения эквивалентны:

1) орторекурсивный ряд Фуръе любого элемента $f \in \mathcal{H}$ по системе $\mathcal{E}$ слабо сходится к разлагаемому элементу;

2) выполнено равенство $G\left(H^{-1} G\right)=G$.

Аналогично, из лемм 2 и 4 может быть получен критерий сильной сходимости ОРР в терминах матрицы Грама. Кроме того, этот критерий может быть приведен к виду, близкому к критерию слабой сходимости. Более формально, имеет место следующая теорема.

Теорема 3. Следующие утверждения эквивалентны:

1) орторекурсивный ряд Фуръе каждого элемента $f \in \mathcal{H}$ по системе $\mathcal{E}$ сходится $к$ разлагаемому элементу;

2) выполнена система равенств

a) $G\left(H^{-1} G\right)=G$,

б) $(\mathrm{CH}) \mathrm{C}^{\top}=C\left(H \mathrm{C}^{\top}\right)$.

Напомним, что по лемме 4 матрица $C$ может быть определена через $G$.

ДоказАтельство. Сначала докажем, что из утверждения 1) следует 2). По лемме 1 сходимость имеет место для всех $e \in \mathcal{E}$, поэтому имеет место и слабая сходимость; следовательно, по теореме 2 справедливо условие а). Из леммы 4 следует, что

$$
C G=C\left(H+H^{\top}-I\right)=C H+C H^{\top}-C=C H+G-C,
$$

откуда с учетом леммы 3 вытекает соотношение $C H=C$. Поскольку имеют место равенства $(C H) C^{\top}=C C^{\top}$ и $C\left(H C^{\top}\right)=C G=G$, а также $C C^{\top}=G$ по лемме 2, то условие б) доказано.

Докажем теперь, что из утверждения 2) следует 1). Пусть для данной системы $\mathcal{E}$ выполняются условия утверждения 2). По лемме 4 из условия а) следует равенство $C G=G$, откуда, как и ранее, вытекает равенство $C H=C$. Тогда из условия б) и леммы 4 следует, что

$$
G=C G=C\left(H C^{\top}\right)=(C H) C^{\top}=C C^{\top} ;
$$

следовательно, по лемме 2 справедливо утверждение 1$)$.

ЗАмечание. В случае, когда $\mathcal{H}=\mathbb{R}^{2}$, условия сходимости из теорем 1 и 2 напрямую следуют друг из друга. Для доказательства введем несколько обозначений. Через $\varphi_{m, n}$ обозначим ориентированный угол от вектора $e_{m}$ до вектора $e_{n}$. Положим $\alpha_{n}=\sin \varphi_{n, n+1}$, $\beta_{n}=\cos \varphi_{n, n+1}, \gamma_{n}=\prod_{k=1}^{n} \beta_{k}$. В этих терминах условие из теоремы 1 эквивалентно равенству $\lim _{n \rightarrow \infty} \gamma_{n}=0$.

Без ограничения общности будем считать, что $e_{1}$ и $e_{2}$ не коллинеарны. Поскольку орторекурсивное разложение - линейный процесс, а векторы $\left\{e_{n}\right\}_{n=3}^{\infty}$ являются линейными комбинациями $e_{1}$ и $e_{2}$, равенство $C G=G$ эквивалентно равенству $C^{\prime} G^{\prime}=G^{\prime \prime}$, где $C^{\prime}-$ подматрица $C$, состоящая из двух ее первых строк, $G^{\prime}$ - подматрица $G$, состоящая из двух 
ее первых столбцов, $G^{\prime \prime}$ - подматрица $G$, состоящая из $g_{11}, g_{12}, g_{21}, g_{22}$. Так как верхняя строка $C^{\prime}$ всегда имеет вид $(1000 \ldots)$, равенство $C^{\prime} G^{\prime}=G^{\prime \prime}$ эквивалентно тому, что произведения второй строки матрицы $C$ на первый и второй столбец матрицы $G$ равны соответственно $g_{21}$ и $g_{22}$. Используя стандартные тригонометрические формулы и применяя метод математической индукции, нетрудно доказать, что $c_{21}=\beta_{1}, c_{2, k}=\alpha_{1} \lambda_{k-1}$, где $\lambda_{1}=\alpha_{1}, \lambda_{k}=\gamma_{k-1} \alpha_{k}$ при $k \geqslant 2$. Положив $\mu_{n}=\cos \varphi_{1, n+1}, \nu_{n}=\sin \varphi_{1, n+1}$ и пользуясь тригонометрическими преобразованиями, несложно установить, что равенство $C^{\prime} G^{\prime}=G^{\prime \prime}$ эквивалентно двум равенствам: $\sum_{k=1}^{\infty} \lambda_{k} \mu_{k}=0$ и $\sum_{k=1}^{\infty} \lambda_{k} \nu_{k}=1$.

Осталось доказать, что последние два равенства эквивалентны равенству $\lim _{n \rightarrow \infty} \gamma_{n}=0$. Складывая тождества $\lambda_{n} \nu_{n}=\gamma_{n-1} \alpha_{n} \nu_{n}$ и $\gamma_{n} \mu_{n}=\gamma_{n-1} \beta_{n} \mu_{n}$, пользуясь тригонометрическими формулами и суммируя результаты, получим, что $\sum_{k=1}^{n} \lambda_{k} \nu_{k}=1-\gamma_{n} \mu_{n}$. Аналогично, $\sum_{k=1}^{n} \lambda_{k} \mu_{k}=\gamma_{n} \nu_{n}$. Так как $\mu_{n}^{2}+\nu_{n}^{2}=1$, эквивалентность имеет место.

Автор выражает искреннюю благодарность своему научному руководителю и рецензенту за ценные замечания и рекомендации.

\section{СПИСОК ЦИТИРОВАННОЙ ЛИТЕРАТУРЫ}

[1] Т. П. Лукашенко, Вест. Моск. ун-та. Сер. 1. Матем., мех., 2001, № 1, 6-10. [2] В. В. Галатенко, Изв. РАН. Сер. матем., 69:1 (2005), 3-16. [3] Р. Кук, Бесконечные матрицы и пространства последовательностей, Физматлит, М., 1960.

\section{А. В. Политов}

Московский государственный университет

им. М. В. Ломоносова

E-mail: antonpolitov-2008@ya.ru
Поступило

12.09.2011

Исправленный вариант

21.12.2011 